Supporting Information

\title{
Self-healable Magnetic Structural Color Hydrogels
}

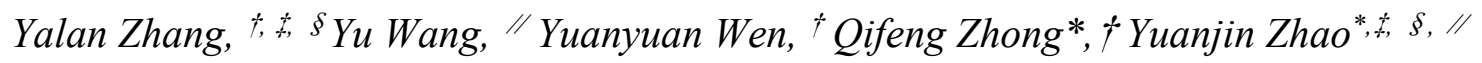

† Department of pharmaceutical engineering, School of engineering, China

Pharmaceutical University, Nanjing 211198, China

$\$$ Department of Clinical Laboratory, Nanjing Drum Tower Hospital, Clinical College of

Xuzhou Medical University, Nanjing 210008, China

$\S$ Department of Clinical Laboratory, The Affiliated Drum Tower Hospital of Nanjing

University Medical School, Nanjing 210008, China

" State Key Laboratory of Bioelectronics, School of Biological Science and Medical

Engineering, Southeast University, Nanjing 210096, China

* E-mail: zhong@cpu.edu.cn.
* E-mail: yjzhao@seu.edu.cn. 


\section{Supporting Figures:}
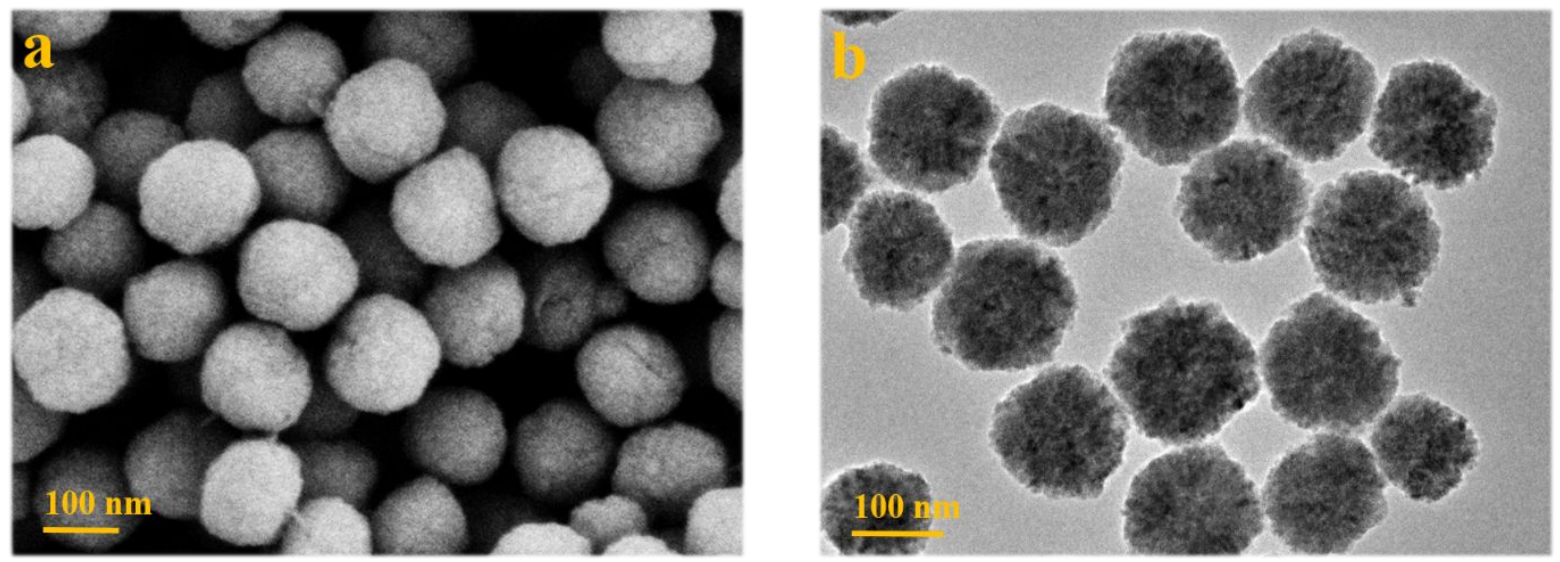

Figure S1. Electron microscopy of magnetic nanoparticles. (a) SEM images and (b) TEM images of the naked magnetic nanoparticles. The scale bars are $100 \mathrm{~nm}$.
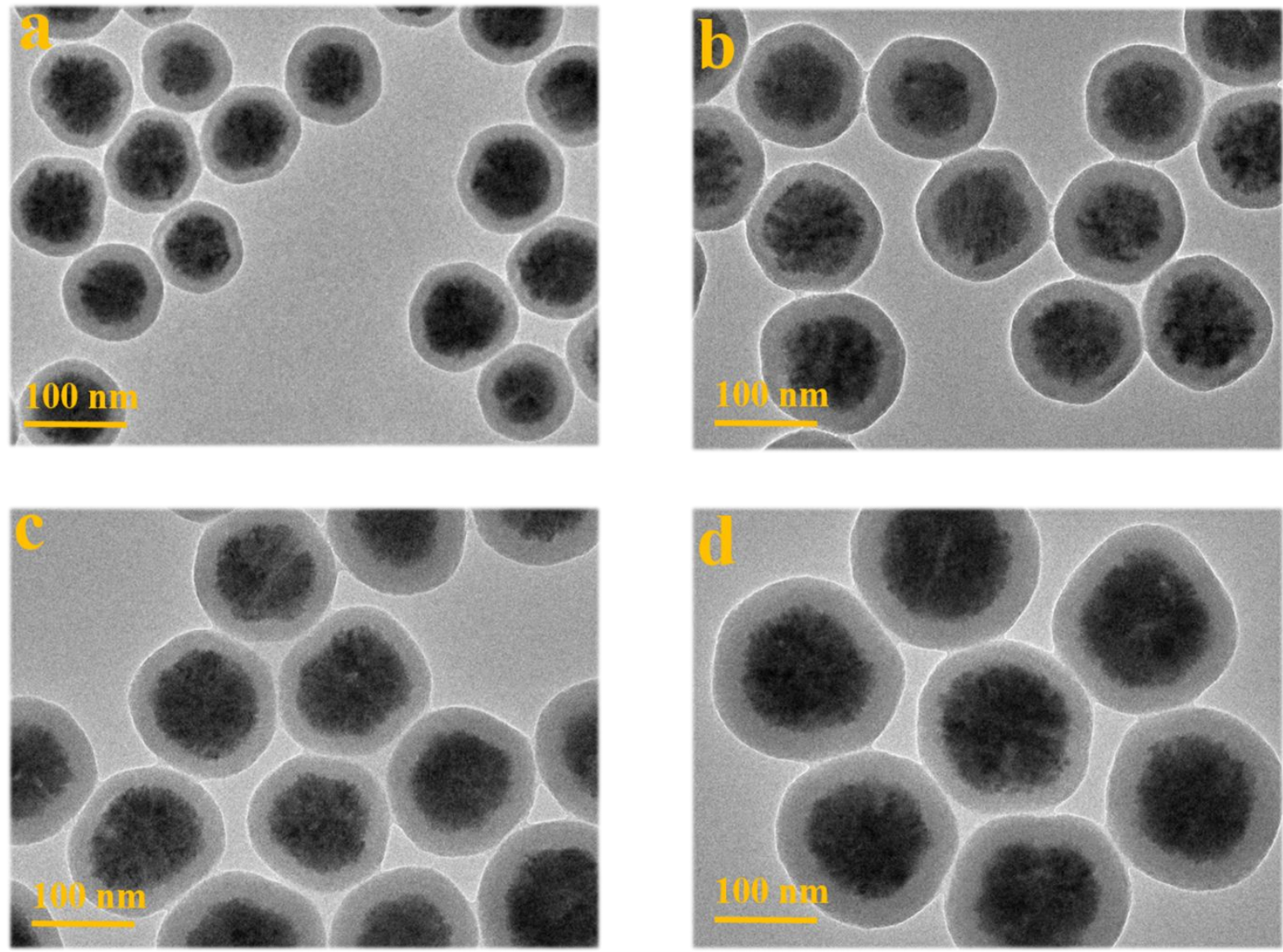
Figure S2. Magnetic nanoparticles with core-shell structure with different particle sizes. (a-d) Sizes distribution and SEM images of $\mathrm{Fe}_{3} \mathrm{O}_{4} @$ PSSMA @ $\mathrm{SiO}_{2}$ nanoparticles by using different amounts of water $(50 \mu \mathrm{L}, 40 \mu \mathrm{L}, 30 \mu \mathrm{L}, 20 \mu \mathrm{L})$. The scale bars are $100 \mathrm{~nm}$.

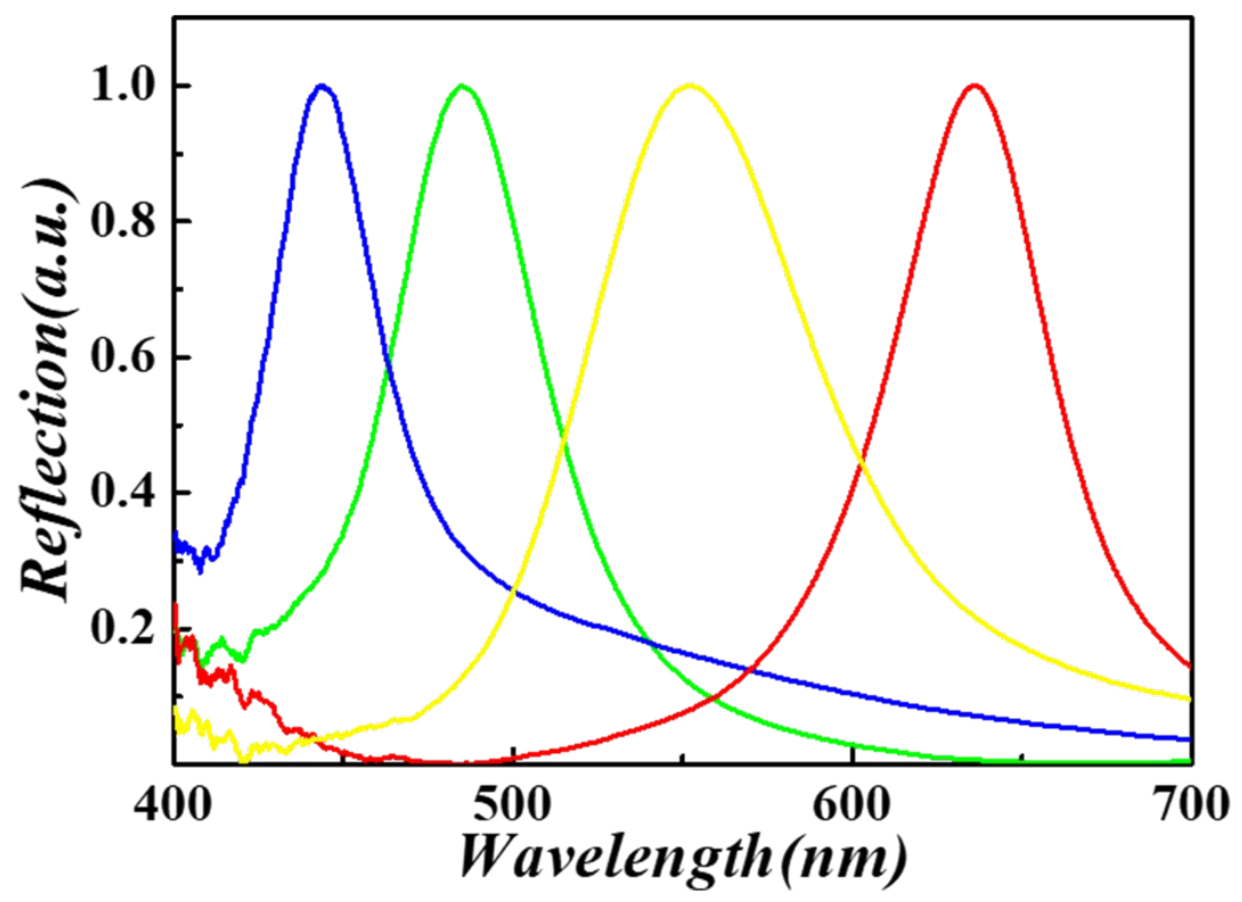

Figure S3. Corresponding refection peaks of different color hydrogels films with magnetic nanoparticles with different particle sizes. 


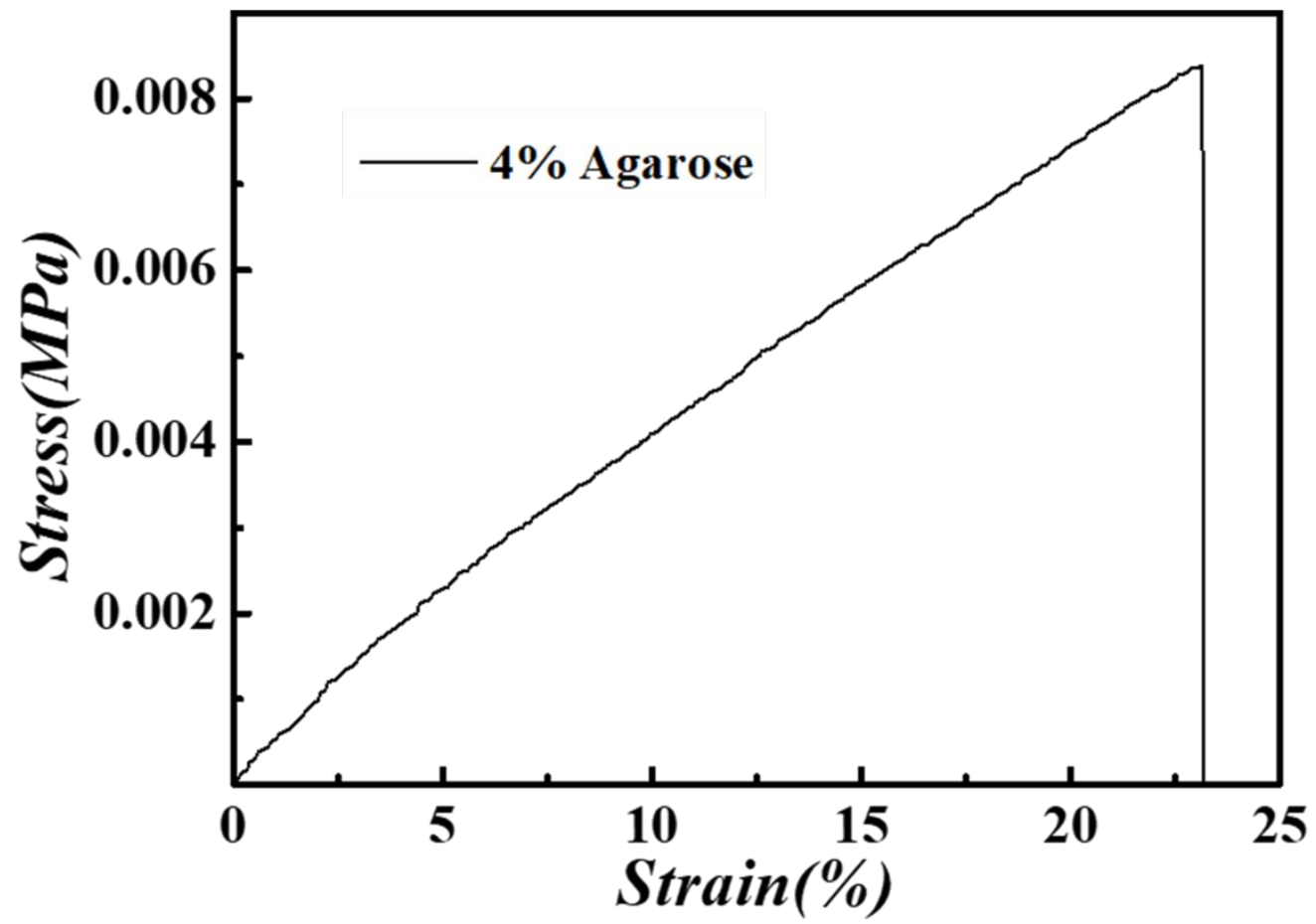

Figure S4. Mechanical strength properties of agarose at 4\% concentration.
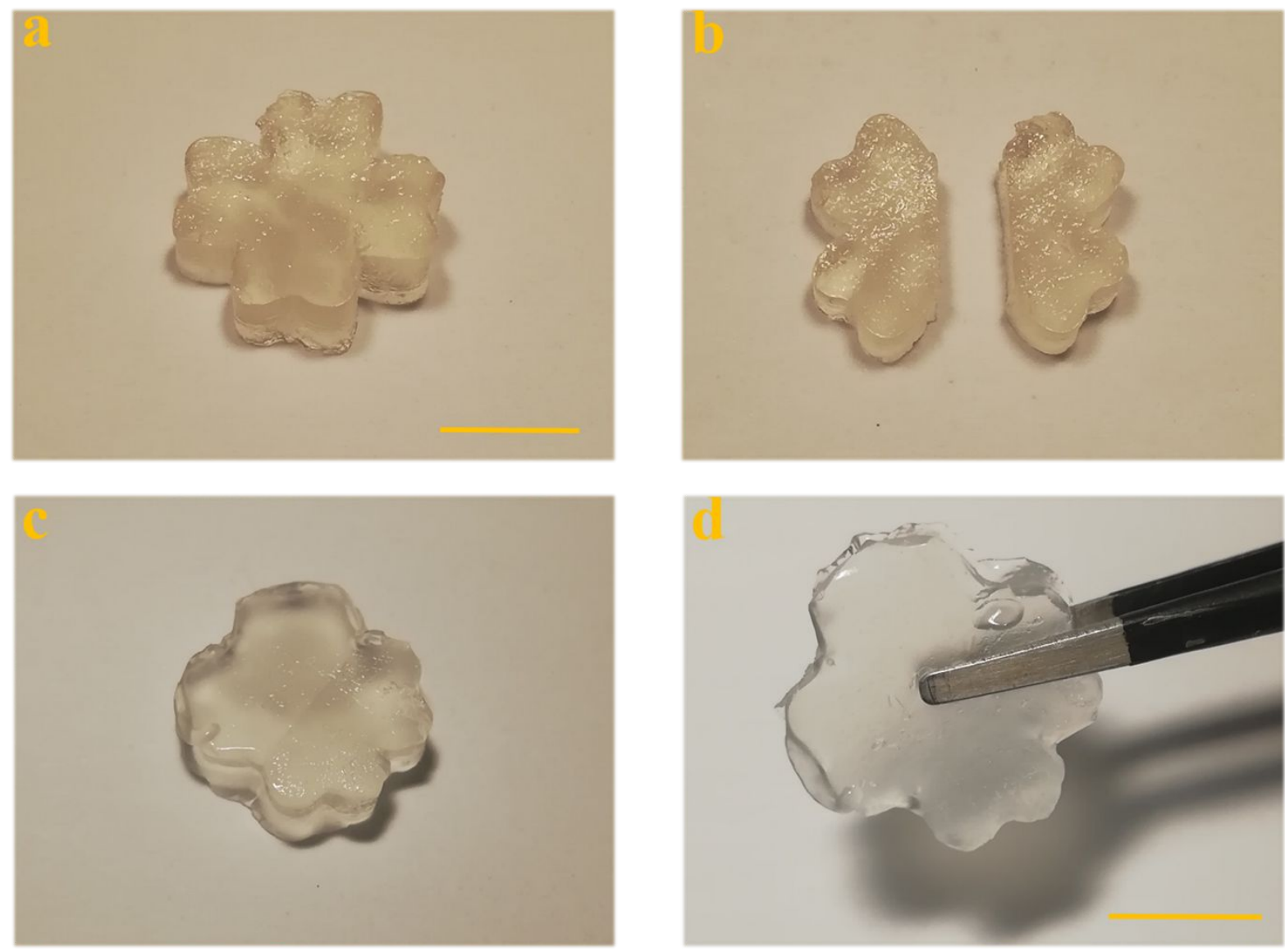

Figure S5. Self- healable process of composite hydrogels. The initial state (a), the cut state (b), 
the heated healing state (c) and the integrity of healed hydrogel. The scale bars are $1 \mathrm{~cm}$.
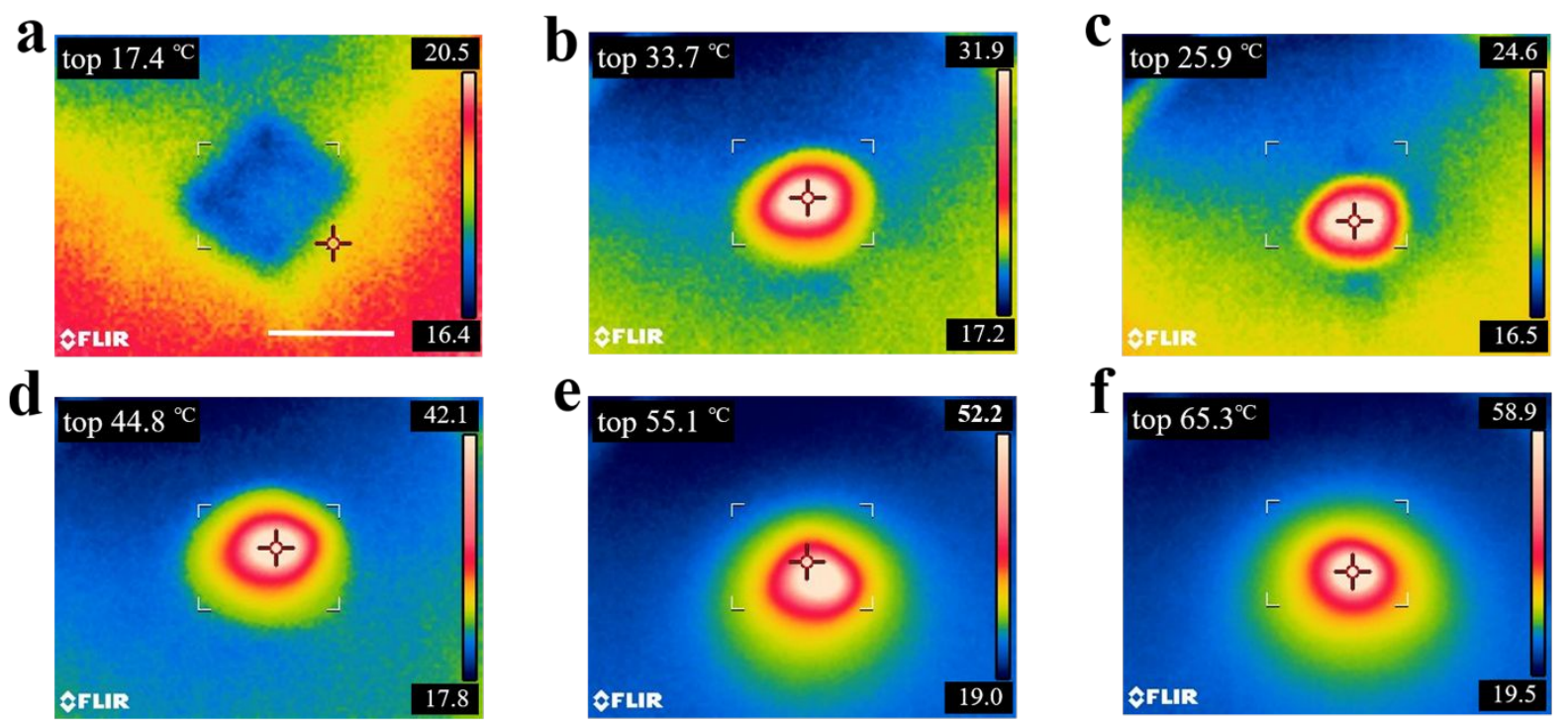

Figure S6. The Infrared response diagram of composite hydrogels. $(a, b)$ The real-time Thermal images of the $80 \mathrm{mg} / \mathrm{mL}$ magnetic nanoparticles in composite hydrogels films under $6 \mathrm{~W}$ of NIR power at distance of $10 \mathrm{~cm}$. The scale bar are $1 \mathrm{~cm}$.
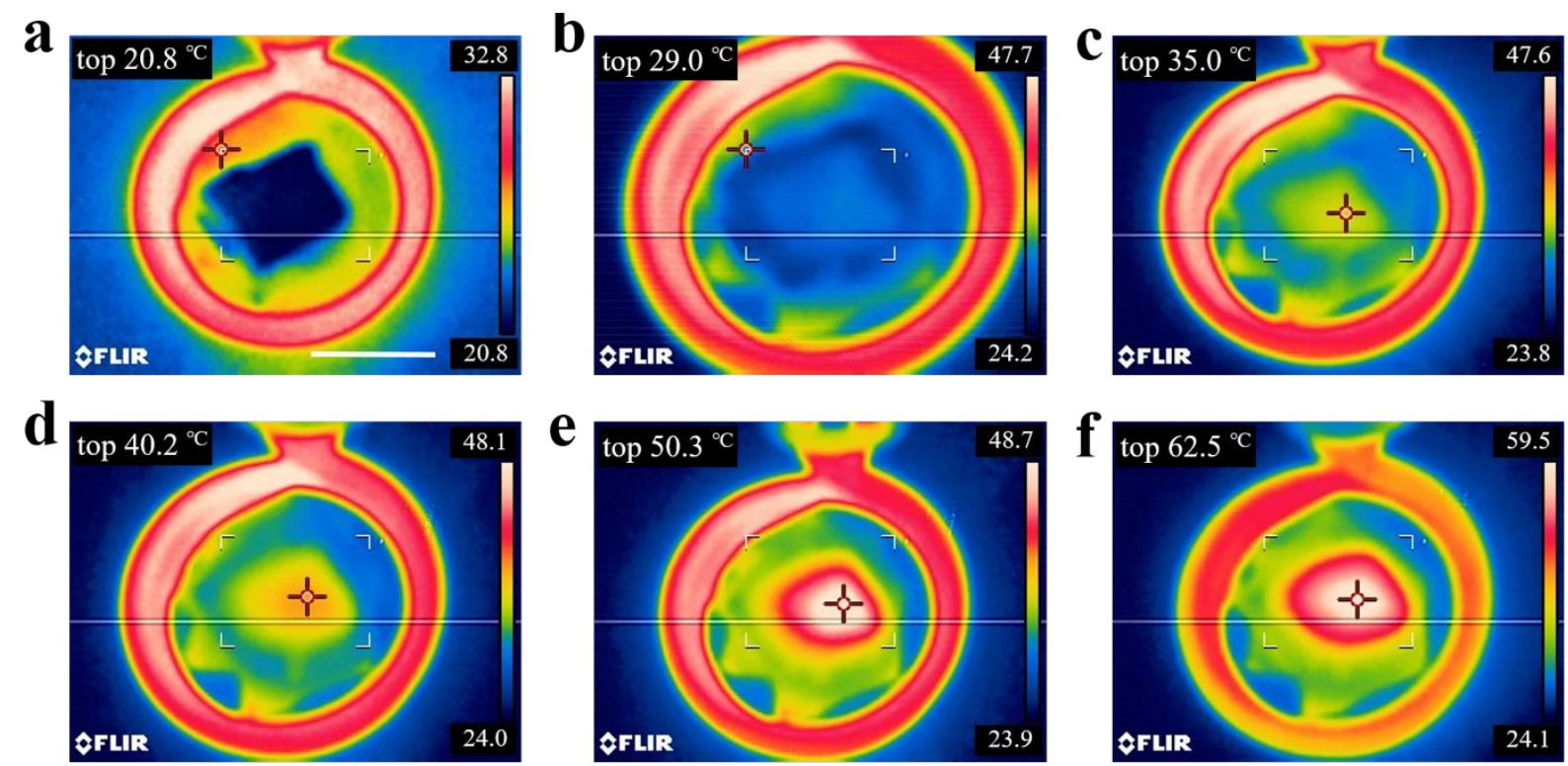

Figure S7. The alternating magnetic fields response diagram of composite hydrogels. (a,b) The real-time Thermal images of the $80 \mathrm{mg} / \mathrm{mL}$ magnetic nanoparticles in composite hydrogels films under 15.8 A of alternating magnetic fields. The scale bar are $1 \mathrm{~cm}$. 


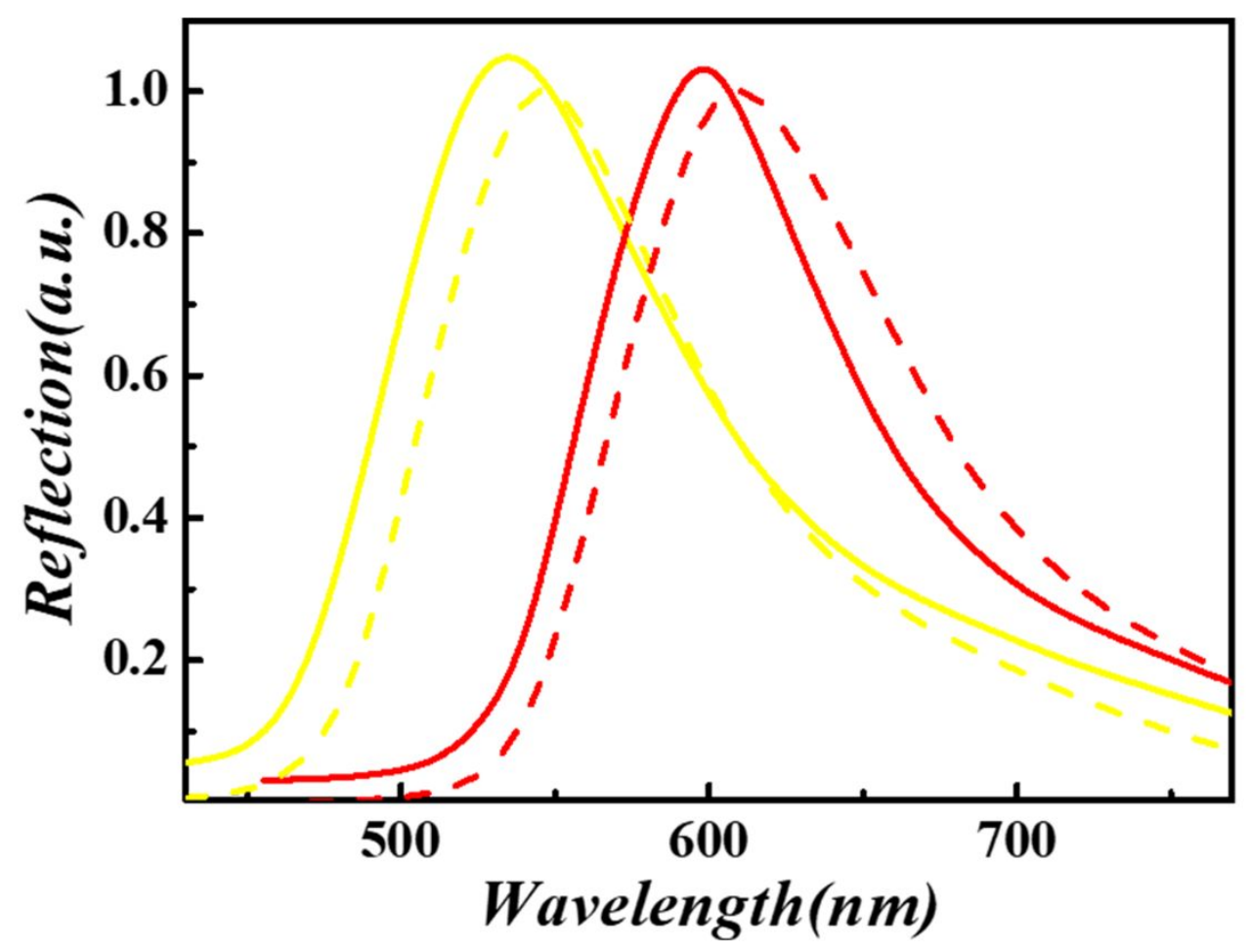

Figure S8. Optical images of two kinds of the magnetic healable structural color hydrogel films and corresponding reflectance spectra.
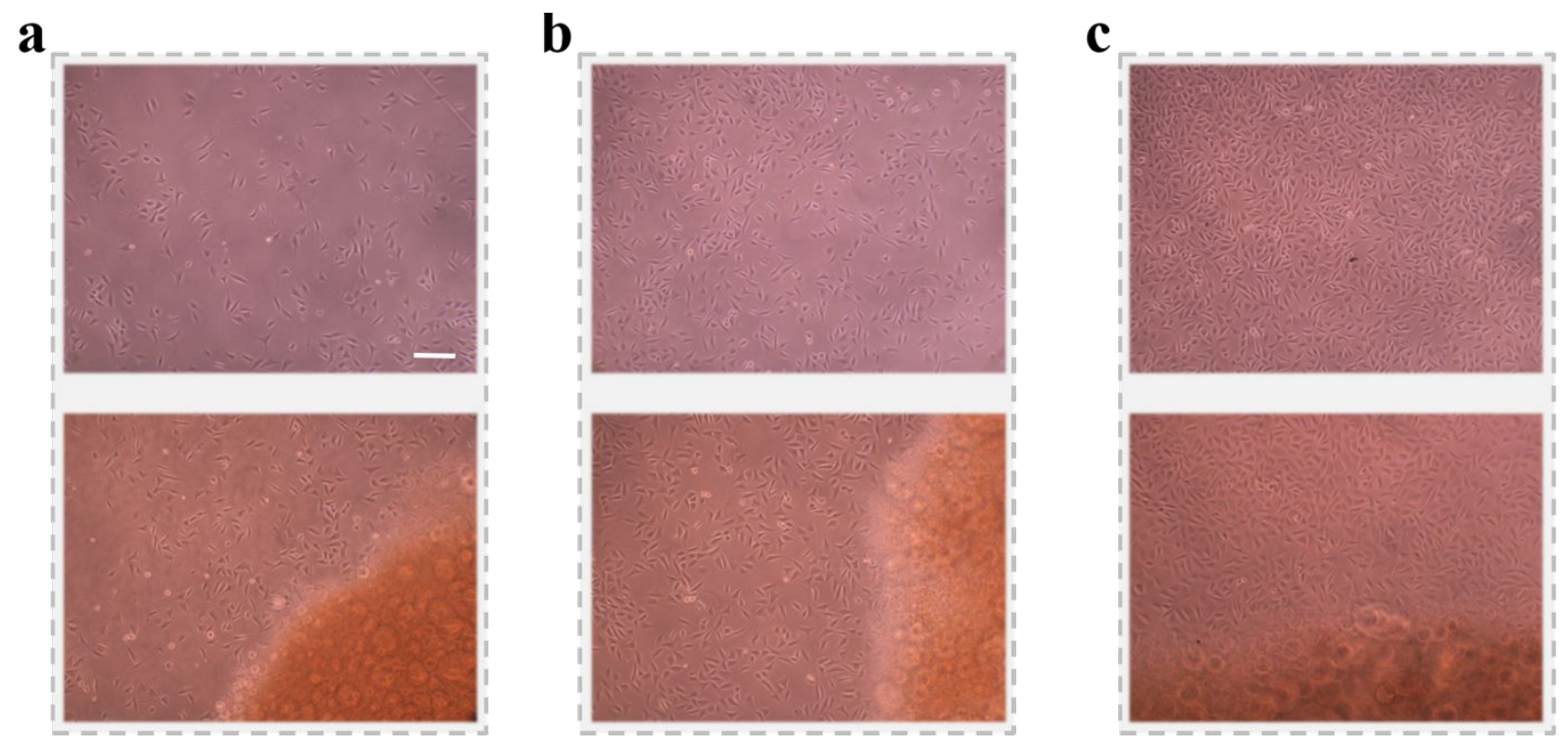

Figure S9. The process of the composite hydrogels was co-cultured with 3 T3 cells. The optical images of the composite hydrogels co-cultured with 3T3 cells for 1days (a), 2 days (b) and 3days (c). The scale bar are $20 \mu \mathrm{m}$. 